\title{
High-resolution Imaging of Reconstituted Protein-DNA Complexes Using Phase Plate Electron Cryo Microscopy
}

\author{
Sara Sandin ${ }^{1,2}$, Radostin Danev ${ }^{3}$, Juergen M. Plitzko ${ }^{3,}$ Eugene Chua Yue Dao $^{1,2}$, Vinod Kumar \\ Vogirala $^{1,2}$, Andrew See Weng Wong ${ }^{1,2}$ \\ 1. School of Biological Sciences, Nanyang Technological University, Singapore \\ 2. NTU Institute of Structural Biology, Nanyang Technological University, Singapore \\ 3. Department of Molecular Structural Biology, Max Planck Institute of Biochemistry, Martinsried, \\ Germany
}

To understand mechanisms involved in gene silencing and activation in higher eukaryotes, detailed knowledge of the structural organization of protein-DNA complexes is needed. Nucleosomes represent the first level of DNA organization in eukaryotes. The Nucleosome Core Particle (NCP) is a 200kDa disk-shaped object, $110 \times 57 \AA$ in diameter, composed of $50 \%$ protein and $50 \%$ DNA. Crystal structures of the NCP show that DNA is wrapped around a protein core, called the histone octamer, in 1.65 superhelical turns [1]. Different histone variants, post-translation modifications of histone proteins, DNA sequences and interacting proteins may directly or indirectly affect chromatin higher-order structure and recruitment of chromatin binding factors [1]. These factors and pathways are misregulated in many different human diseases, such as cancers and viral infections.

In contrast to X-ray crystallography, single particle analysis and electron cryo microscopy (cryoEM) do not require protein crystallization for 3D structure determination. Many NCP-binding proteins and DNA sequences may prevent crystal packing. Therefore, single particle analysis offers the potential to determine novel structures of NCP-complexes that are challenging to crystallize. Thanks to recent advances in instrumentation, in particular the development of direct electron detectors, we now reach near-atomic resolution in cryoEM. However, the molecular weight of the NCP is close to the lower limit suitable for single particle analysis. 200kDa objects are challenging objects for cryoEM imaging due to the inherently low contrast of small complexes.

Here we have used the Volta phase plate [2], a hole-free phase plate, to increase contrast of NCP particles in vitrified ice. Figure 1 shows a comparison of cryoEM images recorded with and without the volta phase plate. The analysis shows that the phase plate provides a contrast enhancement that allows individual nucleosomes and DNA strands to be clearly identified as compared to non-phase plate cryoEM images. This is particularly valuable for detecting disk-view orientations of NCP particles in ice. Next we collected a larger dataset comprising 500 images for single particle analysis. The data was recorded close to focus using a semiautomatic data collection setup with two focus regions. The plate position and correction of astigmatism was done manually. Using this setup, we obtained 400 images of high quality as judged by manual inspection. Next we picked 35,000 particles for 3D structural analysis. After 2D and 3D classification, the best 27,000 particles were selected for refinement. Gold-standard Fourier Shell Correlation shows that phase plate images were of sufficient quality to obtain a nearatomic single particle reconstruction of the NCP. Large protein side-chains and the DNA phosphate backbone can be clearly recognized in the EM density map. The NCP structure presented here represents one of two low molecular weight structures obtained by phase plate cryoEM to date [3]. Future developments in software are required to integrate phase plate changes and astigmatism correction with automatic single particle data collection. [4] 


\section{References:}

[1] K Luger, ML Dechassa, DJ Tremethick, Nat Rev Mol Cell Biol. 13(7) (2012), p. 436-47.

[2] R Danev et al, Proc Natl Acad Sci U S A. 111(44) (2014), p. 15635-40.

[3] M Khoshouei et al, Nat Commun. 7 (2016), p. 10534.

[4] S Sandin, ECY Dao, VK Vogirala and ASW Wong acknowledge funding from Nanyang Technological University and Singapore Ministry of Education Academic Research Fund Tier 3 [MOE2012-T3-1-001], lead-PI D Rhodes. We thank L Nordenskiöld's group for their kind contribution of histones used in this study. We thank M Khoshouei, W Baumeister, C Davey and SHW Scheres for help and advice.
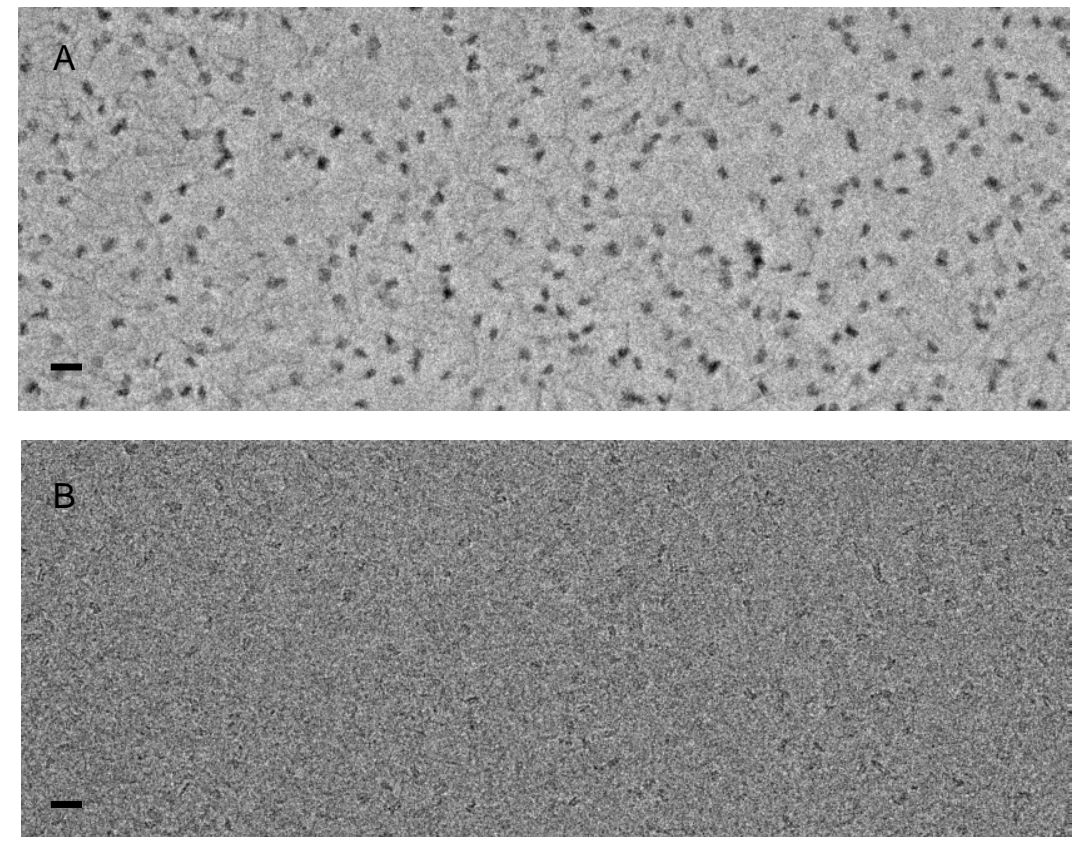

Figure 1. Comparison of cryoEM images of Nucleosome Core Particles and excess DNA, (A) recorded in focus with the volta phase plate and (B) without a phase plate at 2 micrometer underfocus. The scale bar is $20 \mathrm{~nm}$. The images were record at $200 \mathrm{kV}$ on a Tecnai Arctica TEM (FEI), using a dose of 20 electrons/ $\AA^{2}$ and a Falcon2 (FEI) direct electron detector. 